\title{
Evolution and motions of small-scale photospheric structures near a large solar pore
}

\author{
I. Dorotovič ${ }^{1}$, M. Sobotka ${ }^{2}$, P. N. Brandt ${ }^{3}$, and G. W. Simon ${ }^{4}$ \\ 1 Slovak Central Observatory, PO Box 42, 94701 Hurbanovo, Slovak Republic \\ 2 Astronomical Institute, Academy of Sciences of the Czech Republic, 25165 Ondřejov, Czech Republic \\ e-mail: msobotka@asu.cas.cz \\ 3 Kiepenheuer Institut für Sonnenphysik, 79104 Freiburg, Germany \\ e-mail: pnb@kis.uni-freiburg.de \\ 4 Air Force Research Laboratory and National Solar Observatory, Sunspot, NM 88349, \\ and Emeritus Research Services, Santa Fe, NM 87507, USA \\ e-mail: simon@nso.edu
}

Received 17 January 2002 / Accepted 8 March 2002

\begin{abstract}
The analysis of an 11-hour series of high resolution white light observations of a large pore in the sunspot group NOAA 7519, observed on 5 June 1993 at the Swedish Vacuum Solar Telescope, La Palma, Canary Islands, is described. We used a total of 1782 frames, with average time interval of $22 \mathrm{~s}$. Special attention was paid to the evolution of a filamentary region attached to the pore, to horizontal motions around the pore, and to small-scale morphological changes. The filamentary region was observed to change its structure back and forth between penumbra-like filaments and elongated granules. A clockwise rotation of this region around the center of the pore was detected during the whole observing period. This rotation had angular velocities decreasing with time from $7.6^{\circ} h^{-1}$ to $2.7^{\circ} h^{-1}$. Motions inside the filamentary region and around the pore, including penetrations of photospheric granules into the pore, were studied in detail using local correlation and feature tracking algorithms. It was found that the observed filamentary region, although having some typical penumbral features, was different from a normal penumbra.
\end{abstract}

Key words. Sun: activity - Sun: granulation - Sun: sunspots

\section{Introduction}

Sunspots, pores, and small-scale structures in the photosphere have been intensively studied from both theoretical and observational points of view in recent years (see, e.g., the reviews by Sobotka 1997, 1999, and references therein). A detailed investigation of umbral dots and penumbral grains in sunspots is described in Sobotka et al. (1997a,b, 1999a).

Solar pores appear following emergence of a slightly inclined (almost vertical) magnetic field. Sobotka et al. (1999b) analyzed the temporal evolution of fine structures in and around solar pores, especially interactions of pores with surrounding convective motions. They found that motions toward the pore dominate in a $1500 \mathrm{~km}$ zone around the pore boundary, while at larger distances the granules move away from the pore. Such a phenomenon had been observed earlier by Wang \& Zirin (1992), with speeds of $0.5 \mathrm{~km} \mathrm{~s}^{-1}$ and coherence scales of

Send offprint requests to: I. Dorotovič,

e-mail: dorotovic@suh.sk
2000-3000 km. Denker (1998) did not find horizontal motions towards the pore in a series of speckle-reconstructed images, possibly due to the large spatial window used in local correlation tracking. Hurlburt \& Rucklidge (2000) conducted numerical modelling of pores and sunspots as flux elements. In their calculations fluid motions converge towards the flux tube at the surface.

A critical stage in the evolution of sunspots is the formation of a penumbra. Fragments of a penumbra (rudimentary penumbrae) are sometimes seen around pores. Such fragments transform into an ordinary penumbra if the pore evolves into a regular sunspot. Due to the short lifetime of these fragments, it is very difficult to observe this process. Bray \& Loughhead (1964), using whitelight observations, described the formation of a transitory penumbra with a lifetime of almost $3 \mathrm{~h}$. Leka \& Skumanich (1998) investigated the formation of a pore and the appearance of a rudimentary penumbra using data from the Advanced Stokes Polarimeter.

High-resolution observations of sunspots show that penumbral grains move toward the umbra with an 

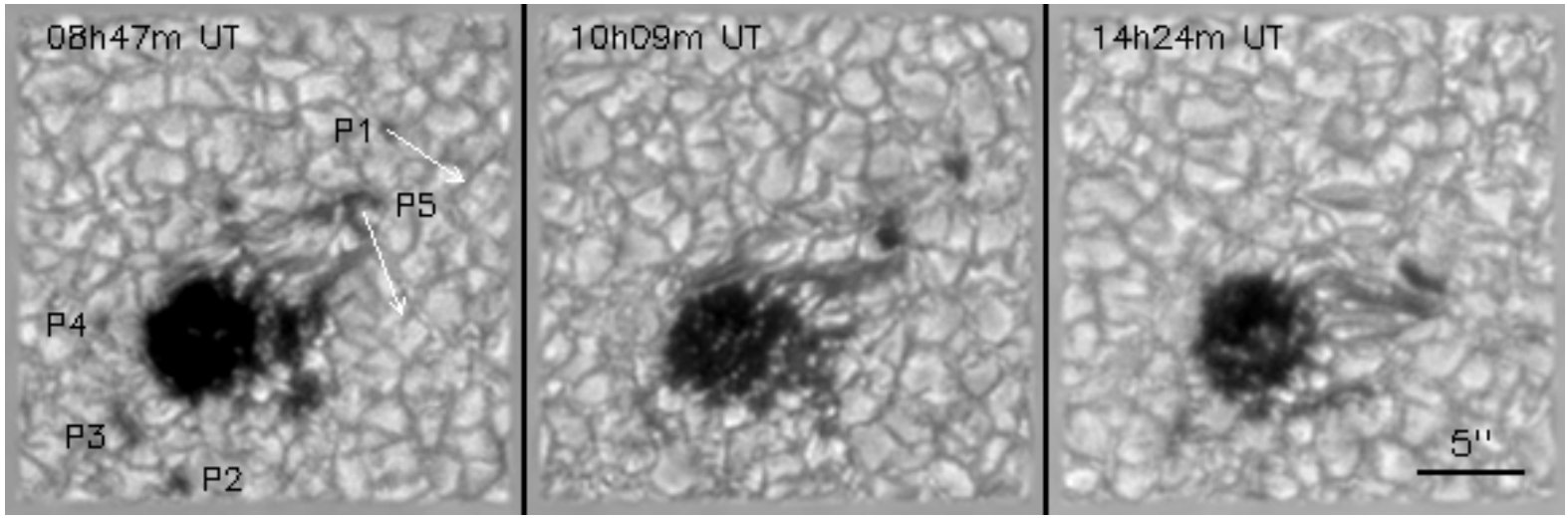

Fig. 1. Large pore in NOAA 7519 with adjacent filamentary region. Distribution of micropores (left panel); white arrows indicate directions of motions of P1 and P5. Examples of the granular (middle panel) and filamentary phases (right panel). The field of view is $24^{\prime \prime} \times 24^{\prime \prime}$.

average speed of $0.3-0.5 \mathrm{~km} \mathrm{~s}^{-1}$ (Muller 1973, 1976; Tönjes \& Wöhl 1982; Wang \& Zirin 1992; MolownyHoras 1994). Sobotka et al. (1999a) found for inward moving penumbral grains a typical proper motion speed of $0.4 \mathrm{~km} \mathrm{~s}^{-1}$ and a median lifetime of $29 \mathrm{~min}$, and for outward moving ones $0.5 \mathrm{~km} \mathrm{~s}^{-1}$ and $22 \mathrm{~min}$. Some penumbral grains may penetrate into the umbra and continue to move as umbral dots (Sobotka et al. 1995). Sobotka et al. (1999b) studied an analogous process that occurs at the pore boundary. They observed a penetration (up to $1^{\prime \prime}$ ) of small granules into the pore with time-averaged speeds ranging from 0.5 to $1.8 \mathrm{~km} \mathrm{~s}^{-1}$.

In this paper we shall concentrate on a filamentary region, so-called because it has a filamentary structure analogous to penumbral filaments, which lies between the upper right quadrant of the large pore and the micropore P5 in Fig. 1. As mentioned above, the formation of a transitory penumbra has only rarely been studied in the past. A first view of our data suggested the initial stage of penumbra formation. The main goal of the present paper is to investigate the evolution of this filamentary structure to determine if it is indeed a rudimentary penumbra, and to study the kinematics of small-scale features in and around the pore.

\section{Observations and data processing}

A detailed description of the observations and data reduction can be found in Sobotka et al. (1997a), so only a brief summary will be given here. The sunspot group NOAA 7519 (see Fig. 1 in Sobotka et al. 1997a) was observed at heliographic position N05, E15 on 5 June 1993 with the Swedish Vacuum Solar Telescope (SVST, cf. Scharmer et al. 1985) at La Palma, Canary Islands. This group reached its maximum area on the date of observation. A $1360 \times 1036$ pixel CCD camera, in connection with a real-time frame selection system, sampled whitelight frames at $\lambda=4680 \pm 50 \AA$ at an image scale of 0'. 125 per pixel. Typical exposure times were 10 to $14 \mathrm{~ms}$. A quad-cell sunspot tracker was locked on a large pore near the sunspot group. This pore is the subject of the present work.

An exceptionally long (11 h) observing run, of persistently high quality seeing, was acquired between $8^{\mathrm{h}} 07^{\mathrm{m}}$ and $19^{\mathrm{h}} 07^{\mathrm{m}}$ UT. After correcting for dark current, flat field, rotation, transparency, and exposure variations, the field of view was reduced to $256 \times 256$ pixels, and, after rigid alignment, further to $192 \times 192$ pixels $\left(24^{\prime \prime} \times 24^{\prime \prime}\right)$, centered on the pore. The superb quality of this time series meant that only $65(3.5 \%)$ of 1868 frames had to be discarded due to poor seeing. The rms granular contrast in the remaining 1803 frames ranged from $6.5 \%$ to $10.6 \%$. The images were then corrected for instrumental profile by means of a Wiener filter and destretched. The frames were interpolated in time to obtain a time series with a constant difference of $22 \mathrm{~s}$ between subsequent frames, and the residual seeing-induced jitter was removed by $k-\omega$ (subsonic) filtering with a cutoff phase velocity of $4 \mathrm{kms}^{-1}$. The first 10 and last 11 frames of the series were unusable due to apodization in the 3-D Fourier filtering process, so that the final time series contained 1782 frames covering the period from $8^{\mathrm{h}} 11^{\mathrm{m}}$ to $19^{\mathrm{h}} 03^{\mathrm{m}} \mathrm{UT}$.

\section{Results}

\subsection{Evolution of the observed region}

In the part of the field of view used for our analysis (see Fig. 1), a large pore, an adjacent filamentary structure, and 5 micropores $\mathrm{P} 1-\mathrm{P} 5$ were present. The area of the large pore showed a linear decrease with time at an average rate of $-0.23 \mathrm{Mm}^{2} \mathrm{~h}^{-1}$ during the whole observing period. We defined this area using the iso-intensity level of 0.6 of the mean intensity of the undisturbed photosphere, after 1". $625 \times 1$ 1". $625(13 \times 13$ pixels $)$ boxcar smoothing. This decrease of the area may be connected with a decrease of the magnetic field strength according to the magnetic field-to-size relation (Martínez Pillet \& Vázquez 1993).

First we studied some general characteristics of the analyzed region. We observed that the structure of the 


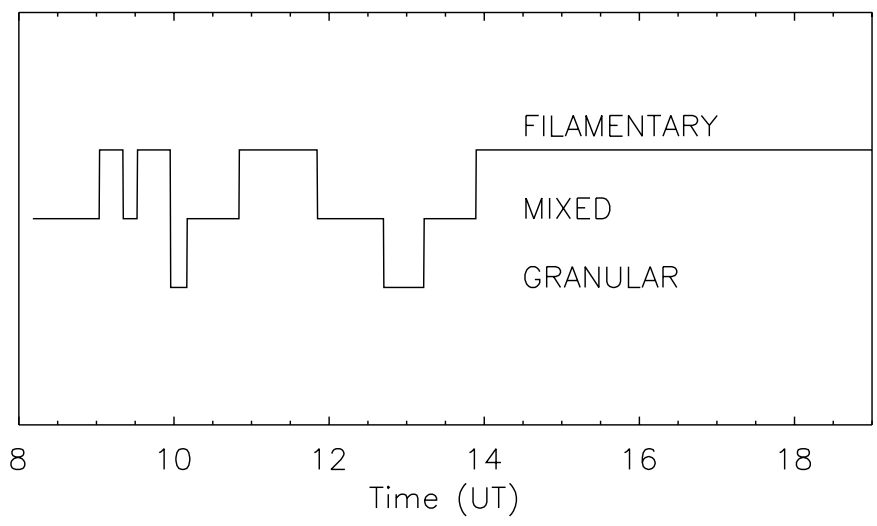

Fig. 2. Time history of prevailing morphological structures in the filamentary region.

region between the large pore and the micropore P5 was not always filamentary, but varied back and forth in time between a filamentary structure and a granular one. During some periods no prevailing filamentary or granular structure was present, but a mixed one (both filamentary and granular). The filamentary structure prevailed for $63 \%$ of the observational period, the granular for $7 \%$, and the mixed one for $30 \%$ (Fig. 2). Each morphological type was accompanied by characteristic horizontal motions (see Sect. 3.2).

A clockwise rotation of the filamentary region around the center of the large pore was observed during the whole 11-hour period. This effect can be clearly seen in Fig. 1. Rotation of the field-of-view due to the altitude-azimuth mounting of the telescope had been eliminated during data reduction, so that the observed rotation values are reliable. This is quickly verified by noting that the micropores P2, P3, and P4 do not show any significant motions during their lifetimes. The position angles of the filamentary region (measured clockwise, with $0^{\circ}$ pointing up in Fig. 1), derived from 45 visual measurements of the position of the micropore P5 located at the end of the filamentary region, are displayed in Fig. 3a together with a cubic-spline fit to the data. The measurements have some uncertainty due to morphological changes of P5. The temporal evolution of the angular velocity (degree $h^{-1}$ ) of the rotation, computed as a derivative of the smooth curve in Fig. 3a, is plotted in Fig. 3b. The total angle of the rotation after $11 \mathrm{~h}$ was $43^{\circ}$. The angular speed of rotation decreased from $7.6^{\circ} h^{-1}$ at the beginning, to $2.7^{\circ} h^{-1}$ at the end, of the observation.

A strong organized flow of granules (pointing to the right at the top of Fig. 4), oriented away from the main spot of the active region, with speeds of $0.6-0.8 \mathrm{~km} \mathrm{~s}^{-1}$, was detected during the first two hours of the observation. Since this occurred when the rotation of the filamentary structure was at its maximum, this flow and the rotation may be related. This organized flow is probably connected not only with processes in the observed region, but also with the evolution of flow fields in the whole active region. No organized flows were detected below the large pore.
The distribution of micropores $\mathrm{P} 1-\mathrm{P} 5$ around the large pore is illustrated in the left panel in Fig. 1. The micropores $\mathrm{P} 1-\mathrm{P} 4$ were seen around the large pore in the first part of the observing period, while $\mathrm{P} 5$, located at the end of the filamentary region, was observed during the whole $11 \mathrm{~h}$. Their motions were measured by means of feature tracking based on correlation between each pair of subsequent frames in a box of size $2 . .5 \times 2 . .5$. The micropores P2, P3, and P4 were nearly stationary with respect to the field of view. P1 and P5 moved coherently with the rotation of the filamentary region around the large pore. The lifetimes and average horizontal speeds of the micropores are listed in Table 1. Since all the micropores were already present at the beginning of the observations, the quoted lifetimes must be considered as lower limits.

Table 1. Lifetimes and horizontal speeds of micropores P1-P5.

\begin{tabular}{lcl}
\hline \hline & Observed lifetime & Avg. horizontal speed \\
\hline P1 & $2^{\mathrm{h}} 15^{\mathrm{m}}$ & $0.42 \mathrm{~km} \mathrm{~s}^{-1}$ \\
P2 & $7^{\mathrm{h}} 04^{\mathrm{m}}$ & no motion \\
P3 & $7^{\mathrm{h}} 02^{\mathrm{m}}$ & no motion \\
P4 & $3^{\mathrm{h}} 30^{\mathrm{m}}$ & no motion \\
P5 & $11^{\mathrm{h}}$ & $0.13 \mathrm{~km} \mathrm{~s}^{-1}$ \\
\hline
\end{tabular}

\subsection{Horizontal motions}

The local correlation tracking algorithm (LCT, November \& Simon 1988) was used to study horizontal motions of granules and small-scale structures in the pore and in the adjacent filamentary region. The reliability of the LCT algorithm has been tested, e.g. by Simon et al. (1995), Roudier et al. (1999) and by Sobotka et al. (1999b). Setting the FWHM of the Gaussian tracking window to 0.5 and the integration time to $15 \mathrm{~min}, 11$ detailed flow maps for various evolutionary phases (filamentary, granular, mixed) of the filamentary region were constructed. An example is shown in Fig. 4. In the granulation around the large pore we observe the well-known diverging flow patterns, with speeds of about $1.0 \mathrm{~km} \mathrm{~s}^{-1}$, from mesogranules and exploding granules. Examples of such outflow centers are at $(44,10),(116,34)$, and $(96,42)$. In addition, the following types of motions were detected: a strong organized flow of granules in the area above the large pore (seen in Fig. 4 in the coordinate range [20-105, 110-125]) described earlier in Sect. 3.1, organized motions in the filamentary region, a penetration of photospheric granules and their fragments into the pore, and a tangential flow of granules and filaments along the border of the pore.

Inside the filamentary region (see the small image inserted in Fig. 4 for easier identification) motions of various features were observed. These included granules of different sizes, bright filaments and their fragments, bright elongated features similar to penumbral grains (hereafter called BEFs), and dark structures. In general, the motions during all evolutionary phases (filamentary, granular, and 

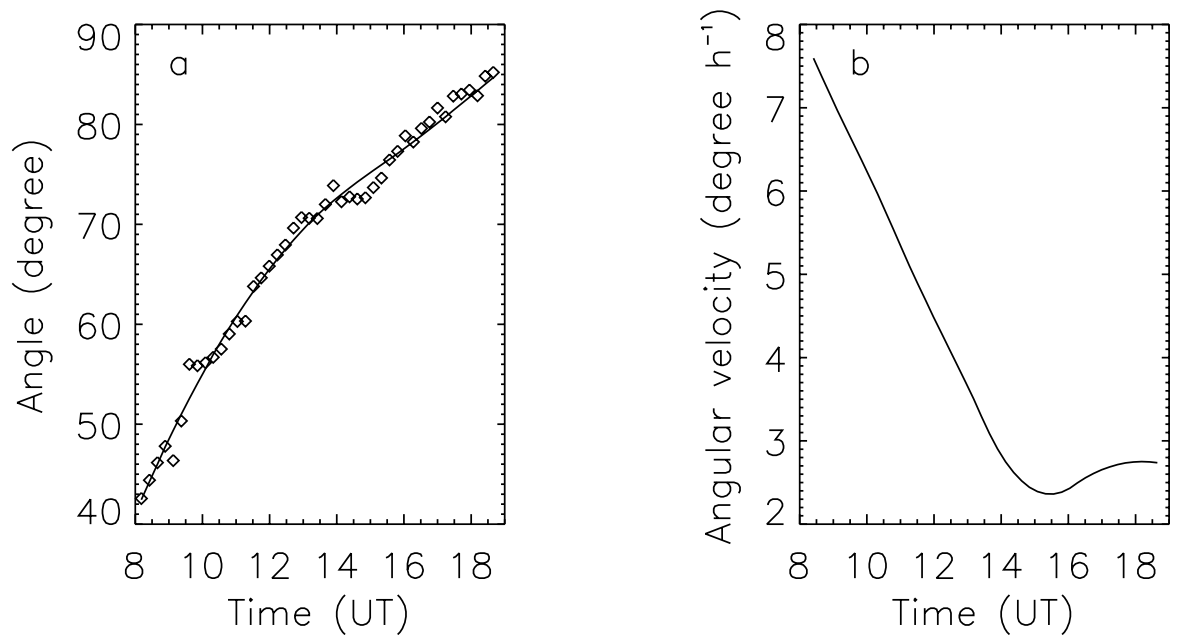

Fig. 3. a) Measured angles of the clockwise rotation of the filamentary region around the center of the large pore vs. time (diamonds) and a cubic-spline fit (solid line) to the data. b) Angular velocity of the rotation vs. time.

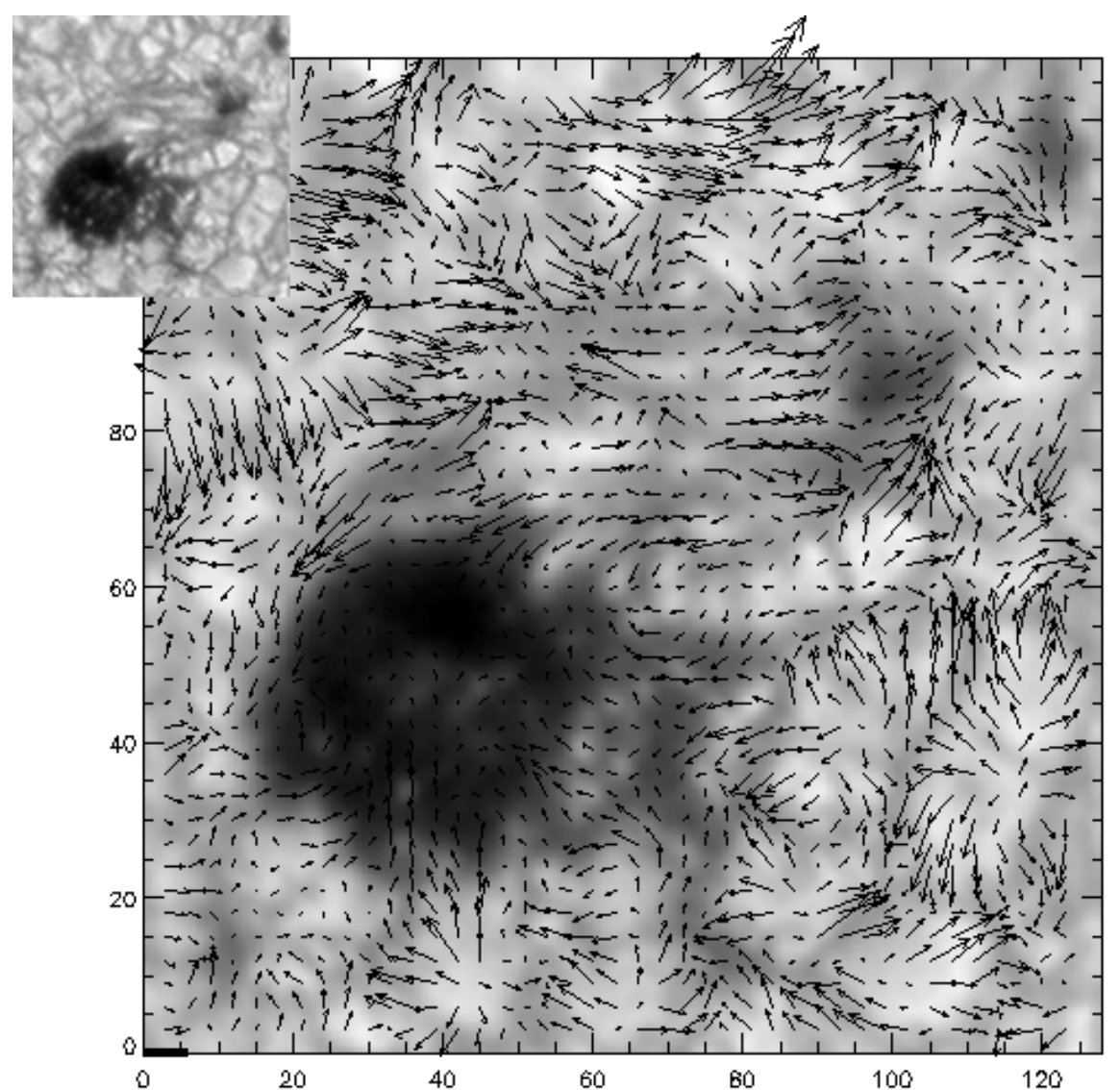

Fig. 4. Horizontal motions in and around the large pore during the filamentary phase $9^{\mathrm{h}} 43^{\mathrm{m}}-9^{\mathrm{h}} 58^{\mathrm{m}}$ UT. The coordinate scale is in pixels ( 1 pixel $=0.125)$, so that the image size is $16^{\prime \prime}$ on a side. The length of the black bar in the lower left corner represents the speed $1 \mathrm{~km} \mathrm{~s}^{-1}$.

mixed) can be described as follows: Near the pore, horizontal speeds were directed toward the pore (inward) and were in the range $0.3-1.0 \mathrm{~km} \mathrm{~s}^{-1}$. The speed decreased with increasing distance from the pore's border. Some granules and BEFs penetrated into the pore. In the middle part of the filamentary structure, the speeds were very small. Far from the pore, motions were usually directed away from the pore (outward) and were in the range 0.5$1.0 \mathrm{~km} \mathrm{~s}^{-1}$, but expanding filaments and granules reached speeds up to $2 \mathrm{~km} \mathrm{~s}^{-1}$. Dark structures moved outward from the pore at approximately the same speed as bright features. In granular phases, the regularity of the outward motions in the region distant from the pore was disturbed due to expansions of elongated granules. In one 
Table 2. Organized motions in the filamentary region.

\begin{tabular}{|c|c|c|}
\hline Phase & Near the pore & Far from the pore \\
\hline Granular & $\begin{array}{l}\text { granules, inward, } 0.5-1.0 \mathrm{~km} \mathrm{~s}^{-1} \\
\text { (some of them penetrate) }\end{array}$ & $\begin{array}{l}\text { granules, outward, } 1.0 \mathrm{~km} \mathrm{~s}^{-1} \\
\text { (expansion of elongated granules) }\end{array}$ \\
\hline Mixed & $\begin{array}{l}\text { filaments, inward, } 0.3-1.0 \mathrm{~km} \mathrm{~s}^{-1} \\
\text { BEFs, inward, } 1.0-1.5 \mathrm{~km} \mathrm{~s}^{-1} \\
\text { granules, inward, } 0.3-0.5 \mathrm{~km} \mathrm{~s}^{-1}\end{array}$ & $\begin{array}{l}\text { filaments, outward, } 0.5-1.0 \mathrm{~km} \mathrm{~s}^{-1} \\
\text { expanding filaments, } 1.5-2 \mathrm{~km} \mathrm{~s}^{-1} \\
\text { granules, outward, } 0.5-1.0 \mathrm{~km} \mathrm{~s}^{-1}\end{array}$ \\
\hline Filamentary & $\begin{array}{l}\text { filaments, inward, } 0.3-1.0 \mathrm{~km} \mathrm{~s}^{-1} \\
\text { BEFs, inward, } 0.5 \mathrm{~km} \mathrm{~s}^{-1}\end{array}$ & $\begin{array}{l}\text { filaments, outward, } 0.5-1.0 \mathrm{~km} \mathrm{~s}^{-1} \\
\text { filaments \& BEFs, inward, } \\
0.3-0.5 \mathrm{~km} \mathrm{~s}^{-1}\end{array}$ \\
\hline
\end{tabular}

particular case of the filamentary phase (from $11^{\mathrm{h}} 27^{\mathrm{m}}$ to $11^{\mathrm{h}} 42^{\mathrm{m}} \mathrm{UT}$ ), some filaments and BEFs far from the pore moved toward the pore with speeds of $0.3-0.5 \mathrm{~km} \mathrm{~s}^{-1}$. This direction of motion is opposite to the usual one. A more detailed listing of the characteristics of motions of various features in different evolutionary phases is shown in Table 2.

Granules penetrated mostly into the bottom part of the pore (image coordinates $[20-45,20-35]$ in Fig. 4). There were nearly no penetrations at the top of the pore; granules moving toward the pore stopped at the border. The pore was darkest at its top; thus we infer that the magnetic field was strongest here (Muglach et al. 1994). This field probably blocked any penetration. The average speed of penetrating features, derived from LCT, was $0.5 \mathrm{~km} \mathrm{~s}^{-1}$ outside the pore and about $0.3 \mathrm{~km} \mathrm{~s}^{-1}$ inside. Near the edges of the filamentary region the speeds were higher: 1.0 and $0.5 \mathrm{~km} \mathrm{~s}^{-1}$. The time-averaged speeds of penetration were also measured using feature tracking with a correlation box of size 1.. $5 \times 1$ 1. 5 . Velocities ranged from 0.9 to $2.0 \mathrm{~km} \mathrm{~s}^{-1}$, with a mean of $1.4 \mathrm{~km} \mathrm{~s}^{-1}$. These values were higher than those derived from LCT, because LCT includes a spatial averaging of velocities of all features present inside the tracking window. Sobotka et al. (1999b) studied the penetration of bright features into a pore using a similar feature tracking method. They obtained average speeds of $2.0 \mathrm{~km} \mathrm{~s}^{-1}$ outside the pore and $0.7 \mathrm{~km} \mathrm{~s}^{-1}$ inside it. A strong flow of granules and fragments of bright filaments, tangential to the border of the pore, can be seen in Fig. 4 along the entire upper left quadrant of the pore in the period $9^{\mathrm{h}} 04^{\mathrm{m}}-11^{\mathrm{h}} 15^{\mathrm{m}}$ UT. The speed decreased with time from 2.0 to $1.0 \mathrm{~km} \mathrm{~s}^{-1}$.

\subsection{Small-scale morphological changes in the filamentary region}

In the evolution of the filamentary region we observed phases when a granular structure dominated and phases with prevailing filamentary structure. Therefore it is essential to pursue the morphological transitions between filaments and granules. Several cases of such transitions were observed. Lifetimes of the filaments and granules ranged from 5 to $30 \mathrm{~min}$, and the typical transition time between a filament and a granule was about $5 \mathrm{~min}$.

An interesting example of a sequence of such morphological transitions is given in Fig. 5, where the indicated times refer to important evolutionary changes of features marked by white arrows: The phenomenon started at $8^{\mathrm{h}} 55^{\mathrm{m}}$ UT with the formation of a bright filament. At $8^{\mathrm{h}} 59^{\mathrm{m}}$ a penumbral-grain-like feature was formed, which in the next 11 minutes expanded first in length $\left(9^{\mathrm{h}} 03^{\mathrm{m}}\right)$ and then in width $\left(9^{\mathrm{h}} 10^{\mathrm{m}}\right)$. At $9^{\mathrm{h}} 15^{\mathrm{m}}$ a granular structure appeared in that location, and reached its maximum in $4 \mathrm{~min}\left(9^{\mathrm{h}} 19^{\mathrm{m}}\right)$. Three minutes later $\left(9^{\mathrm{h}} 22^{\mathrm{m}}\right)$ two narrow filaments appeared, which decayed into a chain of small granules at $9^{\mathrm{h}} 30^{\mathrm{m}}$. A filament then developed from one of the granules $\left(9^{\mathrm{h}} 37^{\mathrm{m}}\right)$, and subsequently a granule formed from a part of the filament $\left(9^{\mathrm{h}} 42^{\mathrm{m}}\right)$. The phenomenon ended with the growth of the granule $\left(9^{\mathrm{h}} 46^{\mathrm{m}}\right)$ and its splitting at $9^{\mathrm{h}} 52^{\mathrm{m}}, 57$ min after the sequence began.

\section{Discussion and conclusions}

Time series of high resolution white-light images were used to study the evolution, horizontal motions, and smallscale morphological changes in the region of a large pore. Attached to this pore a filamentary structure was observed, on which we focused most of our attention. Based on the results presented above, we conclude that:

The observed filamentary region is different from that of a typical penumbra (or a penumbral fragment). The structure of this region exhibits transformations between a filamentary and a granular character. We expect, based on the morphology and kinematics, that this is a region of strongly inclined magnetic field with varying strength, rooted in two magnetic concentrations (the large pore and P5). Unfortunately, we do not have complementary measurements of the magnetic field to decide if their polarities are equal or opposite. Although different from a penumbra, in the filamentary and mixed phases of the evolution, the region shows some similarity to a normal penumbra: inward moving bright features analogous to penumbral grains and outward moving dark structures. The average velocities of BEFs that we measure are comparable to those reported by Sobotka et al. (1999a). 


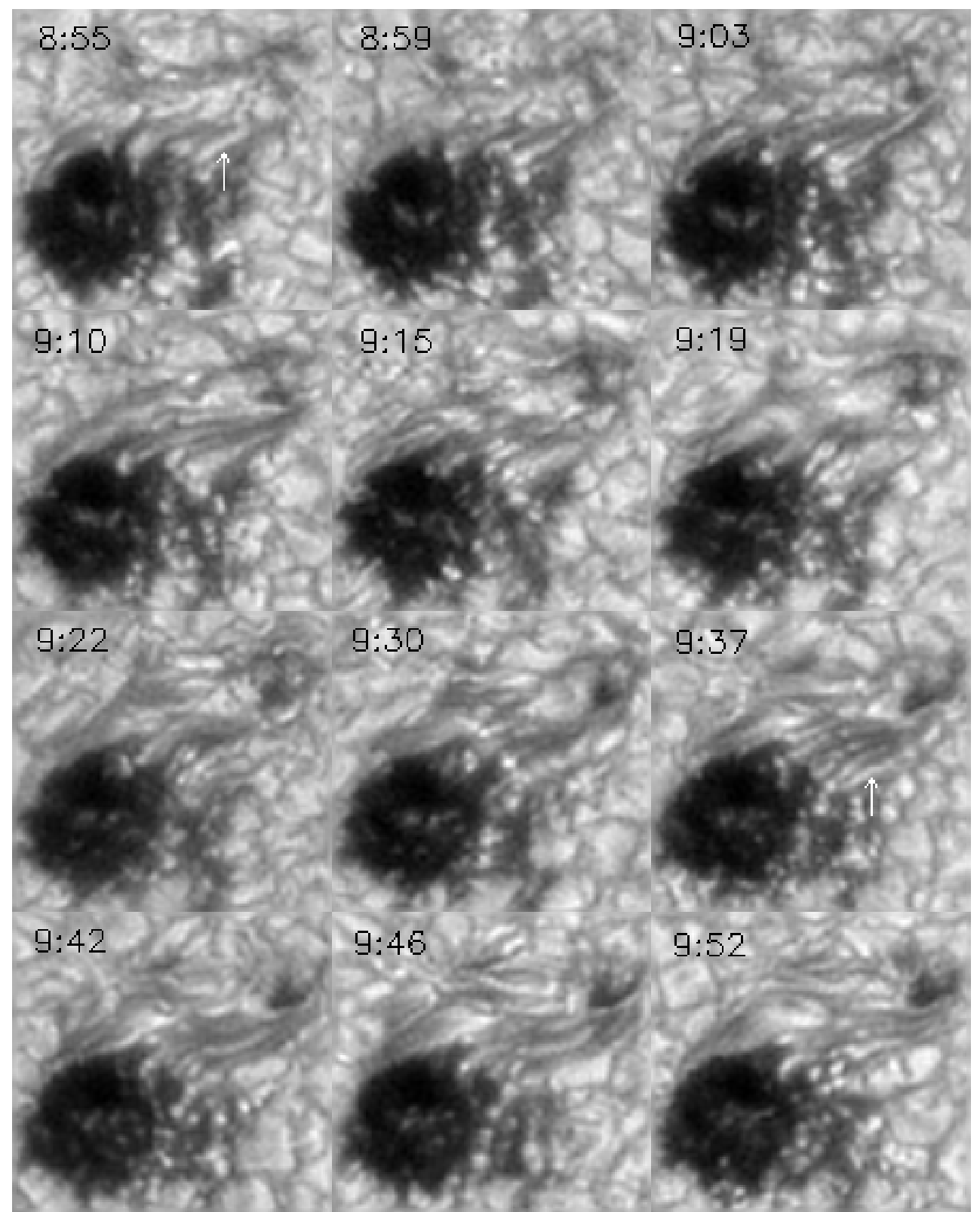

Fig. 5. Transitions between bright filaments and granules. Time is h:mm UT. White arrows point to features undergoing morphological changes. See text for a detailed description.

Perhaps the similarity to a penumbra is due to the combination of a mainly vertical magnetic field in the large pore with a strongly inclined field in the filamentary region.

The region passed through several evolutionary phases with prevailing filamentary or granular structure. We observed individual transitions from bright filaments to granules and vice versa. These changes may reflect variations of field strength or inclination of the assumed nearly horizontal magnetic field. During the first half of the observing run the pore and its surroundings showed an enhanced activity: quick changes between prevailing morphological structures, a fast rotation of the filamentary region, a presence of micropores, a strong organized flow above the pore, and a strong counterclockwise tangential flow in the upper left quadrant of the pore. In the second half of the observation the filamentary structure of the region became stabilized, its rotation was slower, the micropores mostly disappeared, the area of the large pore decreased, and a flow pattern typical of the quiet photosphere prevailed in the surrounding granulation. These activity changes are probably related to the evolution of flow fields in the whole active region in which this pore is imbedded.

The rotation of sunspots about an axis perpendicular to the solar surface has been observed earlier, e.g. by Kučera (1982) who measured the angular velocity of a medium-size spot to vary from $+3^{\circ} h^{-1}$ to $-3^{\circ} h^{-1}$ over 2 days, and recently by Zhao et al. (2001, http://sohowww.nascom.nasa.gov/hotshots/) who discussed a sunspot that rotated about $200^{\circ}$ in less 
than 3 days, which corresponds to an average rotation rate of about $3^{\circ} h^{-1}$. The very high angular velocity of $7^{\circ} h^{-1}$ seen at the beginning of our observing period has not been reported previously, and suggests a rapid evolution of the active region.

In general, during the 11-hour period the observed region was evolving from an active to a stable configuration, presumably including the stabilization of the horizontal magnetic field near the large pore. This is consistent with the evolution of the whole active region NOAA 7519, which reached its maximum area on the day of observation. It remains an open question whether the filamentary region would have developed further into a regular penumbra if the whole active region had continued to grow.

Acknowledgements. We acknowledge the support by G. Hosinsky, R. Kever, G. Scharmer, and W. Wang during the observations at La Palma, and by $\mathrm{R}$. Shine for the prereduction of the data. This work was accomplished under Grants A-3003903 and 205/01/0658 by the Grant Agency of the Academy of Sciences of the Czech Republic (ASCR) and the Grant Agency of the Czech Republic, and under the Key Project K-2043105 of the ASCR. I.D. gratefuly acknowledges the hospitality of the Instituto de Astrofísica de Canarias (IAC). The SVST is operated on the island of La Palma by the Royal Swedish Academy of Sciences at the Spanish Observatorio del Roque de los Muchachos of the IAC.

\section{References}

Bray, R. J., \& Loughhead, R. E. 1964, Sunspots (Chapman \& Hall)

Denker, C. 1998, Sol. Phys., 137, 215
Hurlburt, N. E., \& Rucklidge, A. M. 2000, MNRAS, 314, 793

Leka, K. D., \& Skumanich, A. 1998, ApJ, 507, 454

Kučera, A. 1982, Bull. Astron. Inst. Czechosl., 33, 345

Martínez Pillet, V., \& Vázquez, M. 1993, A\&A, 270, 494

Molowny-Horas, R. 1994, Solar Phys., 154, 29

Muglach, K., Solanki, S. K., \& Livingston, W. C. 1994, in Solar Surface Magnetism, ed. R. J. Rutten, \& C. J. Schrijver (Kluwer), 127

Muller, R. 1973, Sol. Phys., 29, 55

Muller, R. 1976, Sol. Phys., 48, 101

November, L. J., \& Simon, G. W. 1988, ApJ, 333, 427

Roudier, T., Rieutord, M., Malherbe, J. M., \& Vigneau, J. 1999, A\&A, 349, 301

Scharmer, G. B., Brown, D. S., Petterson, L., \& Rehn, J. 1985, Appl. Opt., 24, 2558

Sobotka, M. 1997, in 1st ASPE Conference, Advances in the Physics of Sunspots, ed. B. Schmieder, J. C. del Toro Iniesta, \& M. Vázquez, ASP Conf. Ser., 118, 155

Sobotka, M. 1999, in Motions in the Solar Atmosphere, ed. A. Hanslmeier, \& M. Messerotti (Kluwer), 71

Sobotka, M., Bonet, J. A., Vázquez, M., \& Hanslmeier, A. 1995, ApJ, 447, L133

Sobotka, M., Brandt, P. N., \& Simon, G. W. 1997a, A\&A, 328, 682

Sobotka, M., Brandt, P. N., \& Simon, G. W. 1997b, A\&A, 328, 689

Sobotka, M., Brandt, P. N., \& Simon, G. W. 1999a, A\&A, 348, 621

Sobotka, M., Vázquez, M., Bonet, J. A., Hanslmeier, A., \& Hirzberger, J. 1999b, ApJ, 511, 436

Tönjes, K., \& Wöhl, H. 1982, Sol. Phys., 75, 63

Wang, H., \& Zirin, H. 1992 Sol. Phys., 140, 41

Zhao, J., et al. 2001, private communication, http://sohowww.nascom.nasa.gov/hotshots/ 